\title{
Secreción inadecuada de hormona antidiurética secundaria a estesioneuroblastoma
}

\author{
Beatriz Fernández Medina, Laura de la Maza Pereg, Pablo Herguedas Vela, \\ Isabel E. Escuer Núñez, Irene Esparcia Arnedo
}

Servicio de Endocrinología y Nutrición. Hospital Universitario

de Burgos, España.

Fecha de recepción: 10/12/2015

Fecha de aceptación: 2/02/2016

\section{Presentación}

S e presenta un caso de síndrome de secreción inadecuada de ADH (antidiuretic hormone) de origen paraneoplásico secundario a un estesioneuroblastoma o neuroblastoma olfatorio.

La secreción inadecuada de ADH es la causa más frecuente de hiponatremia euvolémica en la práctica clínica. Los criterios diagnósticos ${ }^{(1)}$ son: sodio plasmático menor a $135 \mathrm{mEq} / \mathrm{L}$, osmolaridad plasmática disminuida, volumen extracelular normal, osmolaridad urinaria mayor a $100 \mathrm{0sm} / \mathrm{Kg}$, sodio urinario mayor a $40 \mathrm{mEq} / \mathrm{L}$, ausencia de insuficiencia suprarrenal o hipotiroidismo.

Como síndrome paraneoplásico se ha asociado a carcinoma microcítico de pulmón, mesotelioma, timoma, duodenal, pancreático, gástrico, síndromes mieloproliferativos y tumores cerebrales ${ }^{(2)}$.

En nuestro caso se asocia con un tumor nasofarígeo, el neuroblastoma, un tumor originado en el neuroepitelio olfatorio de la placa cribiforme a nivel del tercio superior del septo nasal y puede extenderse hacia el espacio intracraneal. Predomina en el sexo masculino, y tiene una distribución bimodal en la edad de presentación, (segunda y sexta décadas) ${ }^{(3)}$. Presentan crecimiento lento y las manifestaciones clínicas más frecuentes son obstrucción nasal seguida de epíxtasis. También cefaleas, anosmia y cambios en la visión.

Fenotípicamente se considera intermedio entre una neoplasia neural pura (neuroblastoma, paraganglioma) y un tumor neuroendocrino epitelial. Presenta positividad para la enolasa neuroespecífica, sinaptofisina y cromogranina A. También puede expresar positividad para citoqueratinas, vimentina, CD-56 y S-100.

El grado histopatológico Hyams (I-IV) tiene en cuenta la preservación de la arquitectura lobular, el índice mitótico, polimorfismo nuclear, presencia de matriz fibrilar, rosetas de Homer-Wright y necrosis.

El tratamiento es la cirugía por vía endonasal o abierta, según el grado de extensión, que puede ser combinado con radiote- rapia o incluso quimioterapia. Se atribuye la causa del síndrome tras la resolución del SIADH luego de la extirpación del tumor.

\section{Presentación del caso}

Mujer de 68 años con antecedentes de menoapusia a los 47 años, hernia de hiato, osteoporosis. No tratamiento habitual ni hábitos tóxicos.

Presenta hiponatremia (con valores mínimos de 114 $\mathrm{mEq} / \mathrm{L}$ ) que oscila entre moderada-grave de 10 años de evolución, a veces sintomática, destacando la emesis, que requirió ingreso para estabilización y estudio en varias ocasiones. En la anamnesis no existía historia de polidipsia.

En la exploración se observa un volumen extracelular normal, con sodio en orina de $131 \mathrm{mOsm}$, osmolaridad en sangre de 240 mOsm y en orina de 928 mOsm. Se descartó hipotiroidismo e insuficiencia suprarrenal. Función hepática, renal, metabolismo ácido base y caliemia normales.

Ante el diagnóstico de SIADH y descartados fármacos que pudieran justificar el cuadro, se realizó TAC abdominopélvico en búsqueda de tumor. Únicamente se detectó nódulo suprarrenal de pequeño tamaño no funcionante.

Tras restricción hídrica, ingesta de sal y administración de diurético de asa los niveles de sodio mejoraron sin llegar a normalizarse, por lo que se decide retirar diurético del asa e iniciar tratamiento con Tolvaptán a dosis de $15 \mathrm{mg}$, con el cual se produce importante mejoría de los niveles de sodio y osmolaridad plasmáticos.

Se decide ampliar estudio con resonancia magnética nuclear hipofisaria que describe hipófisis sin alteraciones, detectándose una alteración de la señal, estructura interna y volumen del cuerpo del esfenoides, basiesfenoides, sugestivo de displasia fibrosa. La paciente presenta tos y rinorrea persistentes por lo que se remite al servicio de otorrinolaringología.

En nasofibroscopia se observa tumoración en fosa nasal izquierda, decidiéndose ampliar estudio con TAC con neuronavegador en el que se describe: tumoración en fosa nasal izquierda, de contornos imprecisos que ocupa prácticamente la totalidad del vestíbulo nasal, oblitera el meato medio y rechaza el tabique nasal. Condiciona ocupación secundaria, probablemente por obstrucción el flujo de drenaje, de los senos maxilar, frontal y celdillas etmoidales izquierdas, con remodelación de las paredes óseas del seno maxilar izquierdo. 
Figura. Corte horizontal TAC

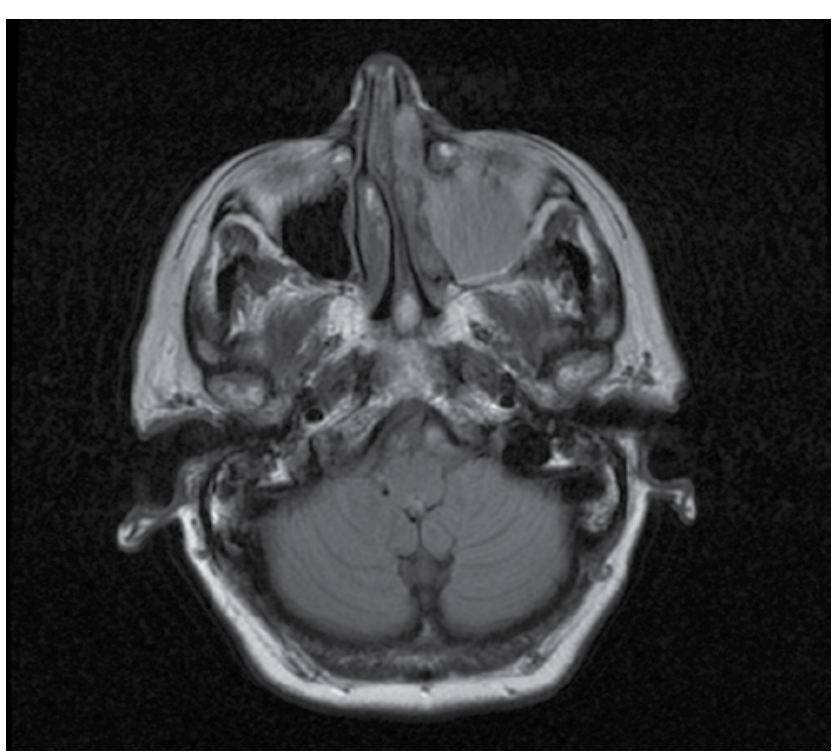

Ante este hallazgo se realiza biopsia con resultado de neuroblastoma olfatorio.

Se procede a diagnóstico de extensión con PET/TC FDG 18 donde se observa depósito patológico de intensidad moderada en fosa nasal izquierda y seno maxilar izquierdo y depósito patológico de intensidad leve-moderada a nivel de adenopatías en los espacios IIA y IIB bilaterales.

Se realiza cirugía endoscópica nasosinusal de fosa izquierda, con necesidad de reintervención por persistencia de restos tumorales. Dada la posibilidad de que el tumor extirpado sea la causa de SIADH se decide retirar tolvaptán, manteniendo la paciente niveles normales de sodio en sangre (137 mEq/L).No existen datos de recidiva en pruebas de imagen de control tras nueve meses de la cirugía.

\section{Discusión}

La asociación de SIADH con tumores de cabeza y cuello se presenta en un 1,5\%-3\%, puede acompañar a tumores de estirpe escamosa, carcinoma neuroendocrino de célula pequeña, carcinoma indiferenciado y sarcoma, se localizan en cavidad nasal y en menor medida en laringe, faringe, seno maxilar y glándulas salivares ${ }^{(5)}$. El desarrollo de SIADH en el estesioneuroblastoma no parece relacionarse con el estadio tumoral ${ }^{(11)}$.

Se han descrito hasta el momento 35 casos de estesioneuroblastoma asociados a SIADH, en 15 de ellos la secreción de ADH se demostró en el tejido. En 26 de ellos el SIADH se resolvió tras el tratamiento del estesioneuroblastoma, 4 murieron a causa del tumor, en 2 persistió el SIADH y 1 presentó reseteo osmótico, o secreción de ADH adaptada a la osmolaridad plasmática pero con un umbral inferior (a diferencia del SIADH paraneoplásico, responden a una sobrecarga hídrica de forma adecuada excretando el agua excedente y aumentando la secreción de $\mathrm{ADH}$ con la restricción hídrica) ${ }^{(4)}$.

En un estudio retrospectivo de 21 pacientes con estesioneuroblastoma, la prevalencia de SIADH fue del 14\%, en la mitad de los casos la hiponatremia precedía a los síntomas del tumor $^{(5)}$. Además, se ha propuesto que la hiponatremia recurrente tras la resección de un tumor secretor de ADH es un marcador de persistencia de la enfermedad ${ }^{(4)}$. De hecho, de los 35 pacientes con ENB asociada a SIADH, 4 han presentado recidiva del tumor y en 3 de ellos la recidiva se asociaba de forma paralela con SIADH tras la resección quirúrgica ${ }^{(8,9,10)}$.

Aunque la cirugía es el tratamiento de elección y también la radioterapia y quimioterapia de forma adyuvante, se han descrito casos de recidiva detectados por Octreoscan, lo que sugiere que esta herramienta podría ser útil para el seguimiento y el uso de análogos de somatostatina para el tratamiento ${ }^{(12)}$.

Se sospecha que la prevalencia de SIADH en el estesioneuroblastoma esté infraestimada, dado que el SIADH subclínico y la hiponatremia leve pueden pasar desapercibidos ${ }^{(4)}$.

De forma característica, el tiempo desde que se detecta el SIADH hasta el diagnóstico del tumor puede llegar a ser muy largo en este tipo de neoplasia, lo que podría indicar el bajo conocimiento de esta asociación hasta el momento debido al reducido número de $\operatorname{casos}^{(7)}$.

Además, se han descrito, aunque de forma poco frecuente, otros síndromes paraneoplásicos de hipersecreción hormonal en el estesioneuroblastoma, tales como productores de ACTH o más raro aún de catecolaminas ${ }^{(11)}$.

\section{Referencias}

1. Ellison DH, Berl T. The síndrome of inappropiate antidiuresis. N Engl J Med. 2007; 356:2064-72.

2. López Hernández MA. Síndromes endocrinos paraneoplásicos. Med Int Mex 2012; 28(5):454-460.

3. Shirley Y. Su, Bell D, Ehab Y. H. Esthesioneuroblastoma, Neuroendocrine Carcinoma, and Sinonasal Undifferenciated Carcinoma: Differenciation in Diagnosis and Treatment. Int Arch Otorhinolaryngol 2014; 18:S149-S156.

4. Kunc M, Gabrych A, Czapiewski P, Sworczak Paraneoplastic syndromes in olfactory neuroblastoma. Contenp Oncol (Pozn) 2015; 19 (1): 6-16.

5. Zohar Y, Talmi YP, Finkelstein Y, Nobel M, Gafter U. Síndrome of inappropiate antidiuretic hormona secretion in cancer of the head and neck. Ann Otol Rhinol Laryngol 1991; 100: 341-4.

6. Gray ST, Holbrook EH, Namj MH, Sadow PM, Curry WT, Lin DT. Syndrome of inappropriate antidiuretic hormone secretion in patients whith olfactory neuroblastoma. Otolaryngol Head Neck Surg 2012; 147: 147-151.

7. Gabbay U, Leider-Trejo L, Marshak G, Gabbay M, Fliss DM. A case and a series of published cases of esthesioneuroblastoma (ENB) in which long-standing paraneoplastic SIADH had preceded ENB diagnosis. Ear Nose Throat J 2013; 92 : E6.

8. Kleinschmidt-DeMasters BK, Pflaumer SM, Mulgrew TD, Lillehei KO. Sinonasal teratocarcinosarcoma ("mixed olfactory neuroblastoma-craniopharyngioma") presenting with syndrome of inappropriate secretion of antidiuretic hormone. Clin Neuropathol 2000; 19: 63-9.

9. Plasencia YL, Cortes MB, Arencibia DM, et al. Esthesioneuroblastoma recurrence presenting as a syndrome of inappropriate antidiuretic hormone secretion. Head Neck 2006; 28: 1142-6.

10. Wade PM Jr, Smith RE, Johns ME. Response of esthesioneuroblastoma to chemotherapy. Report of five cases and review of the literature. Cancer 1984; 53: 1036-41.

11. Senchak A, Freeman J. Case Report: Low-Grade Esthesioneuroblastoma Presenting as SIADH: A Review of Atypical Manifestations. Cases reports in Otolaringology. Volume 2012, Article ID 582180.

12. Freeman SR, Mitra S. Expression of somatostatin receptors in arginine vasopressin hormone-secreting olfactory neuroblastoma-report of two cases. Rhinology. 2005 Mar, 43(1): 61-5. 